\title{
3BDO inhibits the proliferation, epithelial-mesenchymal transition (EMT), and stemness via suppressing survivin in human glioblastoma cells
}

\author{
Zhaotao Wang ${ }^{*}$, Yongping Li ${ }^{*}$, Minyi Liu*, Danmin Chen, Jiajie Lu, Yunxiang Ji ${ }^{\bowtie}$, Zhou Xing ${ }^{\bowtie}$, Yezhong \\ Wang $^{\bowtie}$ \\ Institute of Neuroscience, Department of Neurosurgery, the Second Affiliated Hospital of Guangzhou Medical University, Guangzhou 510260 China \\ *contribute equally \\ $\triangle$ Corresponding author: Yezhong Wang, M.D. Email: wangyezhong@gzhmu.edu.cn Tel: +8613902237122; Zhou Xing, M.D. Email: xingzhou125@126.com Tel: \\ +8613926060228; Yunxiang Ji, M.D. Email: jyx520100@163.com Tel: +8618826267771 The Second Affiliated Hospital of Guangzhou Medical University, People's \\ Republic of China
}

(c) The author(s). This is an open access article distributed under the terms of the Creative Commons Attribution License (https://creativecommons.org/licenses/by/4.0/). See http://ivyspring.com/terms for full terms and conditions.

Received: 2021.08.31; Accepted: 2021.12.29; Published: 2022.01.16

\begin{abstract}
Background: Glioblastoma (GBM) is a tumor of the central nervous system with an extremely poor prognosis. Stemness and EMT play important roles in GBM progression. 3-benzyl-5-((2-nitrophenoxy) methyl) dihydrofuran-2(3H)-one (3BDO), an autophagy inhibitor, has been reported to exert anti-cancer activities on lung carcinoma. However, the effects of 3BDO on GBM remain unknown. Therefore, the purpose of this study was to explore the effects of $3 B D O$ on GBM and to investigate the underlying molecular mechanisms.

Method: CCK-8 experiments and clone formation assays were conducted to determine the level of cell proliferation. Transwell assay was conducted to examine cell migration and invasion abilities. Western blotting and immunofluorescence staining were used to analyze protein expression levels. A xenograft mouse model was used to evaluate the effect of 3BDO in vivo.

Results: We found that 3BDO inhibited U87 and U251 cell proliferation in a dose-dependent manner. Additionally, 3BDO decreased the degree of sphere formation and levels of stemness markers (sox2, nestin, and CD133) in GSCs. 3BDO also inhibited migration and invasion abilities and suppressed EMT markers (N-cadherin, vimentin, and snail) in GBM cells. Moreover, we found that 3BDO downregulated the expression of survivin in both GBM cells (U87, U251) and GSCs. Furthermore, overexpression of survivin decreased the therapeutic effect of $3 B$ DO on EMT, invasion, migration, and proliferation of GBM cells, as well as decreased the stemness of GSCs. Finally, we demonstrated that 3BDO could inhibit tumor growth in a tumor xenograft mouse model constructed using U87 cells. Similar to the in vitro findings, 3BDO decreased the expression of survivin, EMT makers, and the degree of stemness in vivo.

Conclusions: Our results demonstrate that 3BDO can repress GBM both in vitro and in vivo via downregulating survivin-mediated stemness and EMT.
\end{abstract}

Key words: 3BDO, glioblastoma, EMT, GSC, survivin

\section{Background}

Glioblastoma (GBM) is a high-grade glioma with high rates of invasion and raid growth. Despite the development of surgical and medical treatment modalities, patients diagnosed with GBM rarely survive longer than 15 months after diagnosis [1, 2]. Currently, temozolomide (TMZ) is the primary first-line agent used in GBM chemotherapy, but its effectiveness has been marred by drug resistance. Therefore, other agents with higher efficacy need to be urgently identified to manage GBM.

Cancer recurrence and metastasis are often attributed to cancer stem-like cells (CSCs), given their 
capabilities in regenerating tumors by resisting standard chemotherapy and radiotherapy regimens [3-5]. A myriad of cancers has been shown to harbor CSCs, including GBM. CSCs in GBM are known as glioma stem cells (GSCs) and have been shown to be able to undergo multi-lineage differentiation, selfrenewal, and extensive proliferation. Current research has suggested that these GSCs function as pivotal molecules for GBM growth, with their elimination leading to potential GBM growth inhibition [6-8]. It is therefore of great interest to explore the potential use of GSCs as a means of GBM treatment.

The epithelial-mesenchymal transition (EMT) is the mechanism by which normal epithelial cells acquire a mesenchymal phenotype. Cell EMT is the foundation of cancer development, and this process increases the motility of individual cancer cells and allows them to invade across epithelial junctions and extracellular matrices [9, 10]. Therefore, the importance of EMT in GBM is strongly supported. Both in vivo and in vitro studies on GBM have shown that the activation of an EMT-like program results in enhanced malignant cell migration and invasion [11, 12]. Additionally, patients with aggressive GBM and a poor prognosis invariably demonstrate elevated levels of mesenchymal markers, such as $\mathrm{N}$-cadherin, snail, and vimentin $[13,14]$. Therefore, EMT inhibition appears to be a feasible modality of treating GBM.

Survivin is a protein associated with the inhibition of apoptosis and is also known as a baculoviral inhibitor of apoptosis repeat-containing 5 (BIRC5). BIRC5 levels have been found to be significantly elevated in multiple cancers, such as colon cancer, lung cancer, breast cancer, and melanoma $[15,16]$. Similarly, an increasing number of studies have found significantly elevated survivin expression levels in glioma cells, compared with normal central nervous tissue, along with a positive association between survivin expression and pathological glioma grade. Patients with higher levels of survivin have been shown to have a worse prognosis [17]. In addition, increased survivin expression promoted EMT and the stemness process $[16,18]$.

\section{3-Benzyl-5-((2-nitrophenoxy)}

methyl)-dihydrofuran-2 (3H)-one (3BDO) is an mTOR activator that functions as an autophagy inhibitor. For example, it has been reported that 3BDO exposure significantly diminishes the number of autophagosomes in APP/PS1 transgenic mice while simultaneously improving memory function [19]. In addition, 3BDO partially reversed the maturation of Rheb1-deficient neutrophils through mTOR inactivation [20]. Furthermore, accumulating evidence has demonstrated that 3BDO may act independently as an
mTOR modulator. For instance, 3BDO alleviated plaque endothelial cell death and slowed down the establishment of atherosclerosis in mice, but in a manner that was not dependent on autophagy or mTOR activity [21]. 3BDO has also been demonstrated to function as an inflammatory suppressor both in vivo and in vitro [22]. Furthermore, 3BDO has also been shown to inhibit cancer cell growth when administered in combination with DPB [23]. However, it is not well known if 3BDO exerts potential anti-cancer properties in GBM.

This study first dissected the impact of 3BDO on human GBM in vitro. Then, experiments that specifically evaluated EMT and stemness-related properties and their association survivin expression were conducted. The effect of 3BDO on tumor xenograft mice models was also investigated, and it was found to be able to inhibit tumor growth. This study not only identified $3 \mathrm{BDO}$ as a potential agent but also provides a theoretical foundation for a novel approach to GBM treatment.

\section{Materials and Methods}

\section{Chemicals, Reagents, and Antibodies}

$3 \mathrm{BDO}$ was obtained from Selleck. DMSO was used to produce a 3BDO suspension which was kept at $4{ }^{\circ} \mathrm{C}$. Dulbecco's modified Eagle's medium (DMEM) and fetal bovine serum (FBS) were procured from (Grand Island, USA). Antibodies against N-cadherin (\#13116T), nestin (\#33475S), CD133 (\#64326S), vimentin (\#5741T), GAPDH (\#5174) and survivin (\#2802S) were bought from Cell Signaling Technology (Beverly, MA). Abcam (Cambridge, MA) provided antibodies against snail (ab180714) and sox2(ab97959).

\section{Cell Culture}

The glioblastoma cell lines, U87 and U251, were obtained from the Chinese Academy of Medical Sciences (Beijing, China). All cell lines were maintained in DMEM supplemented with $10 \%$ FBS. GSC is a kindly gift from Jia Ouyang from SooChow university, then cultured in GSC medium. Briefly, fresh tumor samples were dissociated into single cells using Accutase (Gibco), filtered and resuspended in GCS medium (Neurobasal, 1× B27, 20 ng/ml EGF, 20 $\mathrm{ng} / \mathrm{ml} \mathrm{bFGF}$, and $1 \times$ GlutaMax). Briefly, GSC populations were isolated by fluorescence-activated cell sorting (FACS) after 6-18 hours recovery in GSC medium. FcR blocking reagent, CD133/2 (293C3)VioBright FITC and isotype control IgG2b-VioBright FITC antibodies (Miltenyi) were used according to the instructions. The GSCs were cultured in GSC medium, which is a neurobasal medium containing 20 $\mathrm{ng} / \mathrm{ml}$ basic fibroblast growth factor (bFGF) (Peprotech, USA), 2\% B27 (Gibco, USA), 1\% 
Glutamine (Gibco, USA), and $20 \mathrm{ng} / \mathrm{ml}$ epidermal growth factor (EGF) (Peprotech, USA). All the above mentioned cells were incubated in a humidified atmosphere with $5 \% \mathrm{CO}_{2}$ at $37^{\circ} \mathrm{C}$.

\section{Cell Viability Assay}

Cells were digested and suspended in a 96-well plate at a density of $4 \times 10^{3}$ cells/well for $24 \mathrm{~h}$. Each well was exposed to various concentrations of 3BDO. After $24 \mathrm{~h}$, the cells were further treated with $10 \mu \mathrm{l}$ of CCK-8 solution and allowed to incubate for an hour at $37^{\circ} \mathrm{C}$. A microplate reader was used to determine cell viability.

\section{Clonogenic Assay}

Cells were suspended in DMEM medium containing $10 \%$ FBS. 500 cells were added to a plate with a diameter of $6 \mathrm{~cm}$. The selected 3BDO concentrations were added, and the cells were cultured for 1 week at $37^{\circ} \mathrm{C}$ in a $5 \% \mathrm{CO}_{2}$ atmosphere until cell colonies were visible. The cells were then rinsed thrice with PBS before fixation with methyl alcohol and stained with crystal violet for $10 \mathrm{~min}$. The number of colonies was counted after three final rinses with PBS. The experiments were performed in triplicate.

\section{Sphere Formation Assay}

Single GSC cells (3000 cells per well) were plated onto a $6 \mathrm{~cm}$ ultra-low attachment plate (Corning) used above containing GSC medium, following 3BDO treatment, as indicated. Cells were cultured under standard cell culture conditions. After 5 days of culture, the number of spheres larger than $50 \mu \mathrm{m}$ was counted under a microscope. The experiment was performed in triplicates, and at least 5 fields of view were evaluated in each replicate.

\section{Cell migration and Invasion Assay}

A Transwell system (Corning, USA) was used to study cell invasion and migration abilities. The upper chamber with $8 \mu \mathrm{m}$ pores was used to house the cells $\left(2 \times 10^{4}\right.$ suspended $200 \mu \mathrm{l}$ of DMEM supplemented with $1 \%$ FBS). The chambers were coated with $100 \mu 1$ of matrigel (B.D. Biosciences, CA, USA) to conduct the migration assays or left uncoated for the invasion assays. $600 \mu \mathrm{l}$ of DMEM supplemented with $20 \%$ FBS was placed in the lower chamber along with various concentrations of 3BDO. The cells were incubated for 24 hours. After this period, the cells were removed from the upper chamber using a cotton swab, and cells in the lower chamber were fixed with ethanol and stained with crystal violet. The number of cells was counted in three random fields viewed at 100x magnification.

\section{Transfection}

Both GSCs and the GBM cell lines were transfected with a survivin gene-containing plasmid to produce cells that overexpressed survivin (GeneCopoeia, Maryland Rockville, USA) using jetPRIME® Versatile DNA transfection reagent (Polyplus Transfection, France). Control cells were transfected with the vector only. All experiments were conducted as instructed by the manufacturer.

\section{Western Blotting Assays}

Xenograft glioblastoma tissue homogenates and cell lysates were used for the western blotting assays. The Pro-prep TM protein Extraction Solution (iNtRON Biotechnology, Korea) was used to extract proteins. $10-12 \%$ sodium dodecyl sulfatepolyacrylamide gel electrophoresis (SDS-PAGE) was performed to separate the component proteins before blotting them onto polyvinylidene difluoride membranes (Merck, KGaA, Darmstadt, Germany). 5\% BSA was used to block unspecific reactions for an hour at room temperature. The membranes were then incubated overnight with specific primary antibodies at $4^{\circ} \mathrm{C}$. Horseradish protein-conjugated secondary antibodies were exposed to the membranes for an hour at room temperature. A Super Signal ECL (Pierce, Rockford, IL, USA) system was used to interpret the results. Samples derived from the same experiment and their gels/blots were parallel processed.

\section{Immunofluorescence}

After the treatments indicated above were conducted, the GSCs were incubated on cell climbing slices coated with poly-lysine for 1 hour. This was followed by immunofluorescence staining in accordance with standard protocols. 1× PBS was first used to rinse the slides thrice before normal goat serum supplemented with $0.3 \%$ Triton X-100 was added and allowed to incubate for an hour at room temperature. Then, specific primary antibodies suspended in $300 \mu \mathrm{l}$ of $5 \%$ BSA were added onto the slides and allowed to incubate overnight at $4^{\circ} \mathrm{C}$. The cells were once again washed thrice with PBS the next morning and exposed to Alexa Fluor 555-conjugated secondary antibody at room temperature for 45 minutes. Then, the slides were once again rinsed thrice and counterstained with DAPI for $10 \mathrm{~min}$, washed, and air-dried. Images were captured under either light or fluorescence microscopy under a confocal microscope.

\section{U87 Xenograft Mouse Model with 3BDO Treatment}

Female BALB/c nude mice were procured from 
the Animal Experiment Center of Southern Medical University (Guangzhou, China). The mice were 6-8 weeks old and were reared under institutional animal care guidelines after the experimental procedures involving animals were approved by the Institutional Animal Care Committee of the Institutional Animal Care of the Second Affiliated Hospital of Guangzhou Medical University. Guidelines for the ethical review of laboratory animal welfare (People's Republic of China National Standard GB/T 35892) and ARRIVE guidelines (https://www.nc3rs.org.uk/arrive-guide line) were also followed [24]. All mice received subcutaneous injections containing cultured U87 cells $\left(5 \times 10^{6}\right.$ cells per mouse) to the dorsum. Calipers were used to assess tumor size in two orthogonal directions. Tumor volume $\left(\mathrm{mm}^{3}\right)$ was derived based on the following formula: $1 / 2 \times$ length $\times$ width $^{2}$. Upon achieving a tumor size of approximately 150 $\mathrm{mm}^{3}$, the mice were either intraperitoneally injected with the vehicle or $3 \mathrm{BDO}(80 \mathrm{mg} / \mathrm{kg} /$ day) ( $\mathrm{n}=5$ mice per group). The body weight and tumor size of the mice were assessed once every 5 days. All tumors were harvested for further analysis after the mice were sacrificed at the end of these experiments.

\section{Statistical analysis}

All data indicated are the average of the results of experiments performed in triplicate and were analyzed using SPSS 20.0 software. An independent T-test was used to perform simple comparisons between 2 groups, and while comparisons between multiple groups were evaluated using one-way analysis of variance, followed by post hoc analysis, using Dunnett's T3 test or Turkey test. A P $<0.05$ was interpreted as a statistically significant result.

\section{Results}

\section{BDO inhibits the proliferation, migration, and invasion of GBM cells}

As shown in Figure 1 A-B, U87 and U251 cell growth were inhibited by $3 \mathrm{BDO}$ in a dose-dependent manner after 24 hours of treatment. Similarly, 3BDO also suppressed the colony formation ability of the cells in a dose-dependent manner (Figure 1C-D). Next, we observed the invasive and migratory abilities of cells treated with 3BDO using transwell assays. In contrast to the untreated groups, the 3BDO treated groups exhibited lower rates of cell invasion and migration in a dose-dependent manner (Figure 1E-H). These in vitro findings strongly suggest that 3BDO inhibits the proliferation, invasion, and migration of GBM cells.

\section{BDO decreases EMT marker expression and inhibits survivin expression in GBM cells}

Exposure to $50 \mu \mathrm{M}$ or $100 \mu \mathrm{M}$ doses of 3BDO for 24 hours resulted in a lower level of $\mathrm{N}$-cadherin, vimentin, and snail expression than the $0 \mu \mathrm{M}$ group (Figure 1I-M). Meanwhile, 3BDO reduced survivin expression levels in a dose-dependent manner in U87 and U251 cells. Our findings indicate that 3BDO decreases the expression of EMT markers and inhibits survivin expression in GBM cells.

\section{BDO inhibits cell growth and downregulates survivin expression and stemness markers in GSCs}

The initiation and progression of GBM have been attributed to the effect of GSCs. It is, therefore, of sound scientific reasoning to pursue GSC as a therapeutic target in GBM. The GSCs were cultured in a sphere-forming medium and were treated with different concentrations of 3BDO for $72 \mathrm{~h}$. As shown in figure $2 \mathrm{~A}-\mathrm{B}, 3 \mathrm{BDO}$ treatment decreased the number of GSC spheres in a dose-dependent manner.

Sox2 is an important marker of GSC stemness. We found that sox2 expression levels decreased as 3BDO concentration increased (Figure 2C-D). Next, western blotting analysis was performed to quantify the expression of stemness markers, such as nestin, CD133, and sox2. The results showed that the aforementioned stemness marker expression levels were downregulated after incubation for $24 \mathrm{~h}$ with the indicated doses of 3BDO. Moreover, we found that 3BDO decreased survivin expression levels in a dose-dependent manner in GSCs (Figure 2E-F). These results suggest that $3 \mathrm{BDO}$ is able to suppress stemness and downregulate survivin levels in GSCs.

\section{Overexpression of survivin decreased the effect of 3BDO on GBM cells and GSCs}

Previous studies have highlighted the role of survivin in EMT. We aimed to confirm whether 3BDO-induced EMT suppression was mediated by survivin inhibition. U87 and U251 cells were artificially induced to overexpress survivin before being subjected to a $24 \mathrm{~h}$ of incubation with either $3 \mathrm{BDO}$ or the vehicle. The proliferation, migration, and invasion abilities of the GBM cells increased in the presence of survivin overexpression (Figure 3A-F). We also found that survivin upregulation increased the expression of EMT markers, including $\mathrm{N}$-cadherin, vimentin, and snail (Figure 3G-J). Furthermore, we found that the overexpression of survivin reduced the inhibitory effects of $3 \mathrm{BDO}$ on GBM cells. 
A
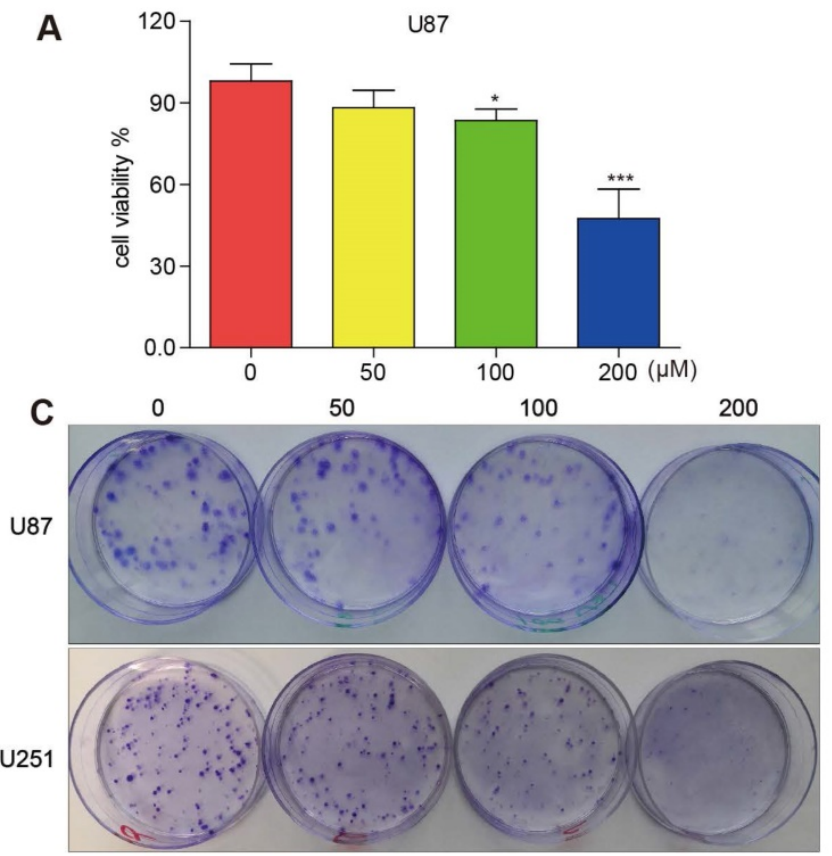

E

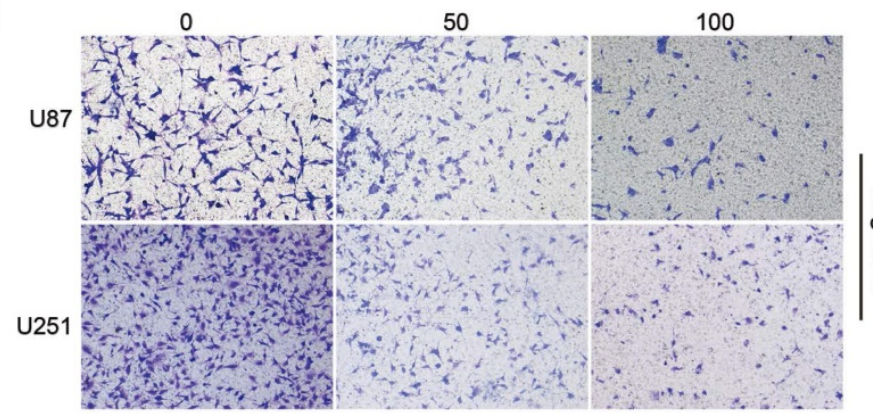

G

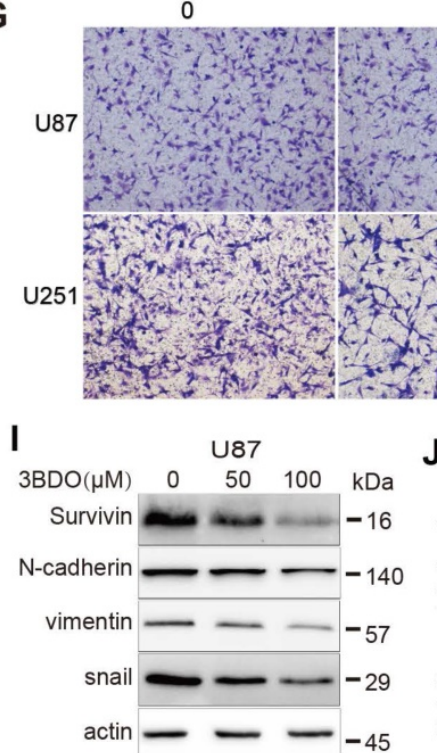

50

100
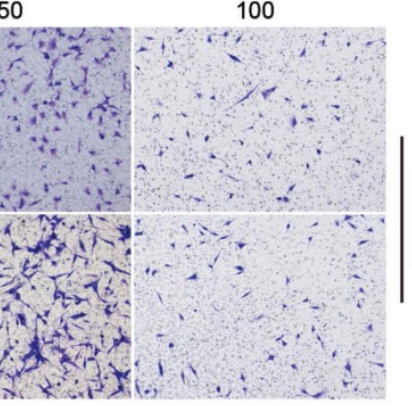

J

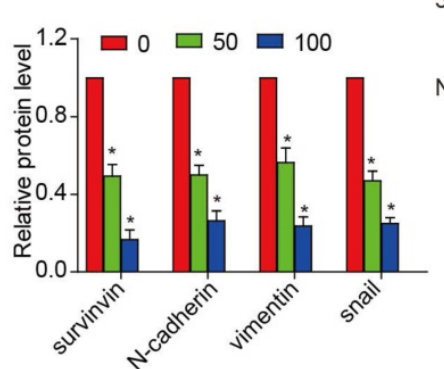

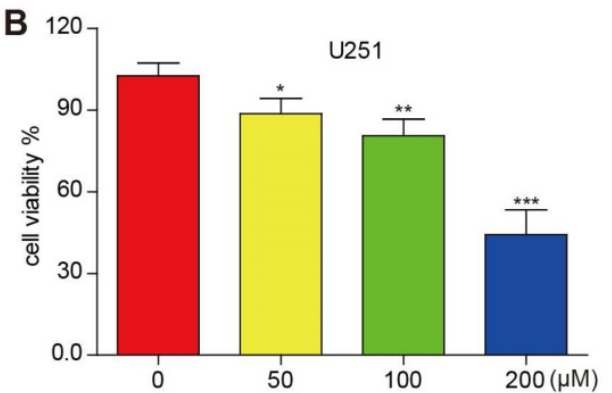

D

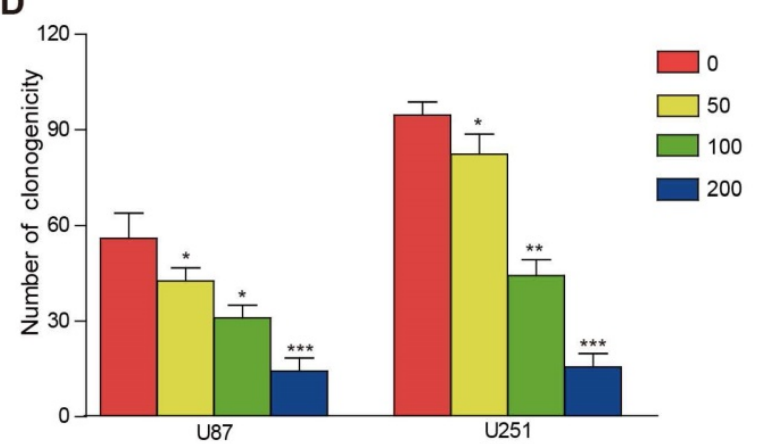

F
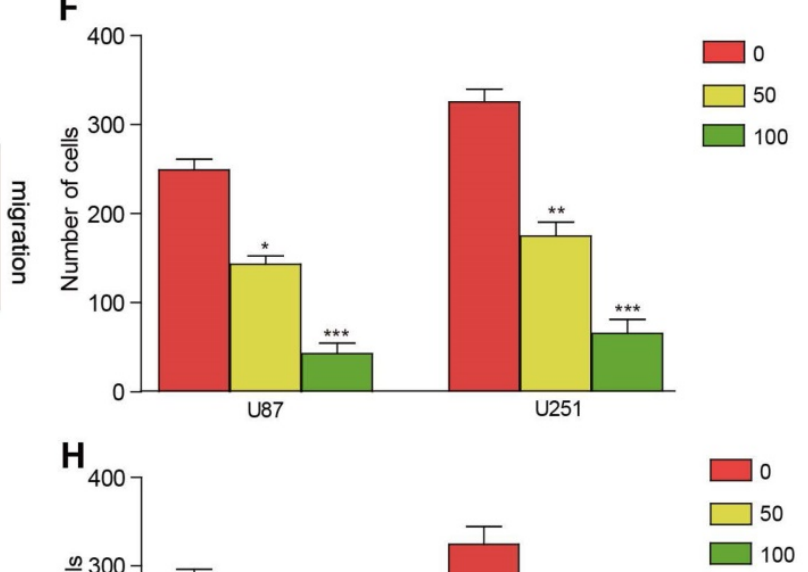

$\mathbf{L}_{3 B D O(\mu M)} \quad 0 \quad \mathrm{U}^{2} 50 \quad 100 \quad \mathrm{kDa} \mathbf{M}$
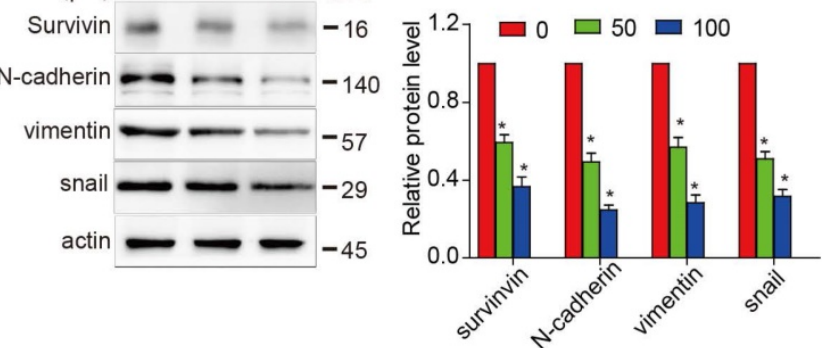

Figure 1. 3BDO diminishes the invasion, migration, and proliferation abilities and the expression of EMT-associated molecules and survivin in GBM cells. (A-B) U87 and U251 cells were treated with 3 BDO for $24 \mathrm{~h}$, followed by tests to confirm cell viability. (C-D) U87 and U251 cells were treated with $3 B D O$ for 7 days before the clonogenic assay was performed. (E-H) GBM cells were incubated with selected 3BDO concentrations for 24 hours and were subjected to a transwell assay to evaluate the degree of cell invasion and migration. (I-M) Western blotting analysis was used to investigate the expression of survivin, and EMT-associated proteins in U87 and U25I cells exposed to selected concentrations of 3 BDO for $24 \mathrm{~h}$, and the gels were analyzed. $n=3$ or $n=4$ in each group. All experiments were performed in triplicate. *, $P<0.05$ compared with the control $(0 \mu \mathrm{M})$. 
A

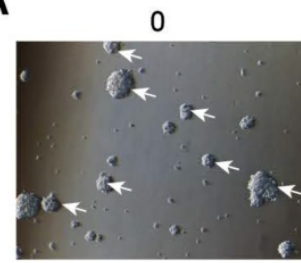

50

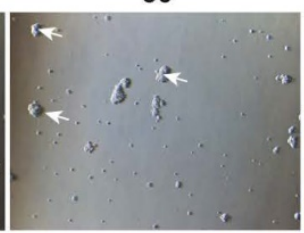

100

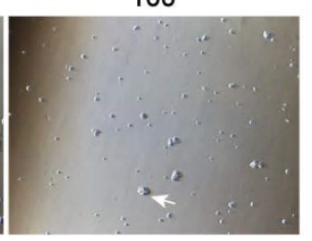

B

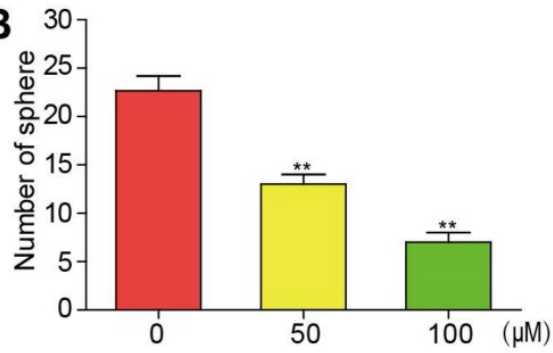

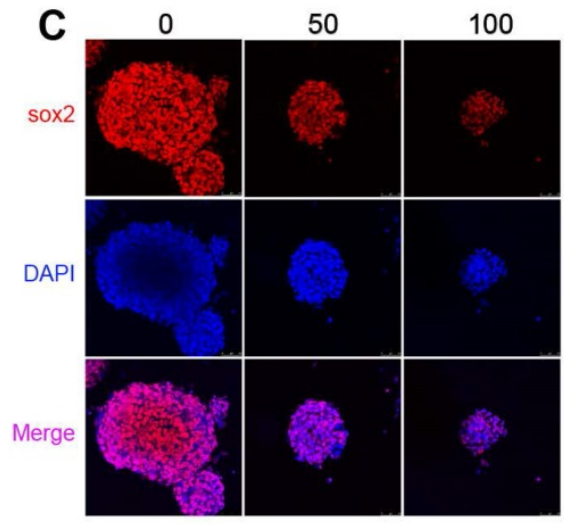

D
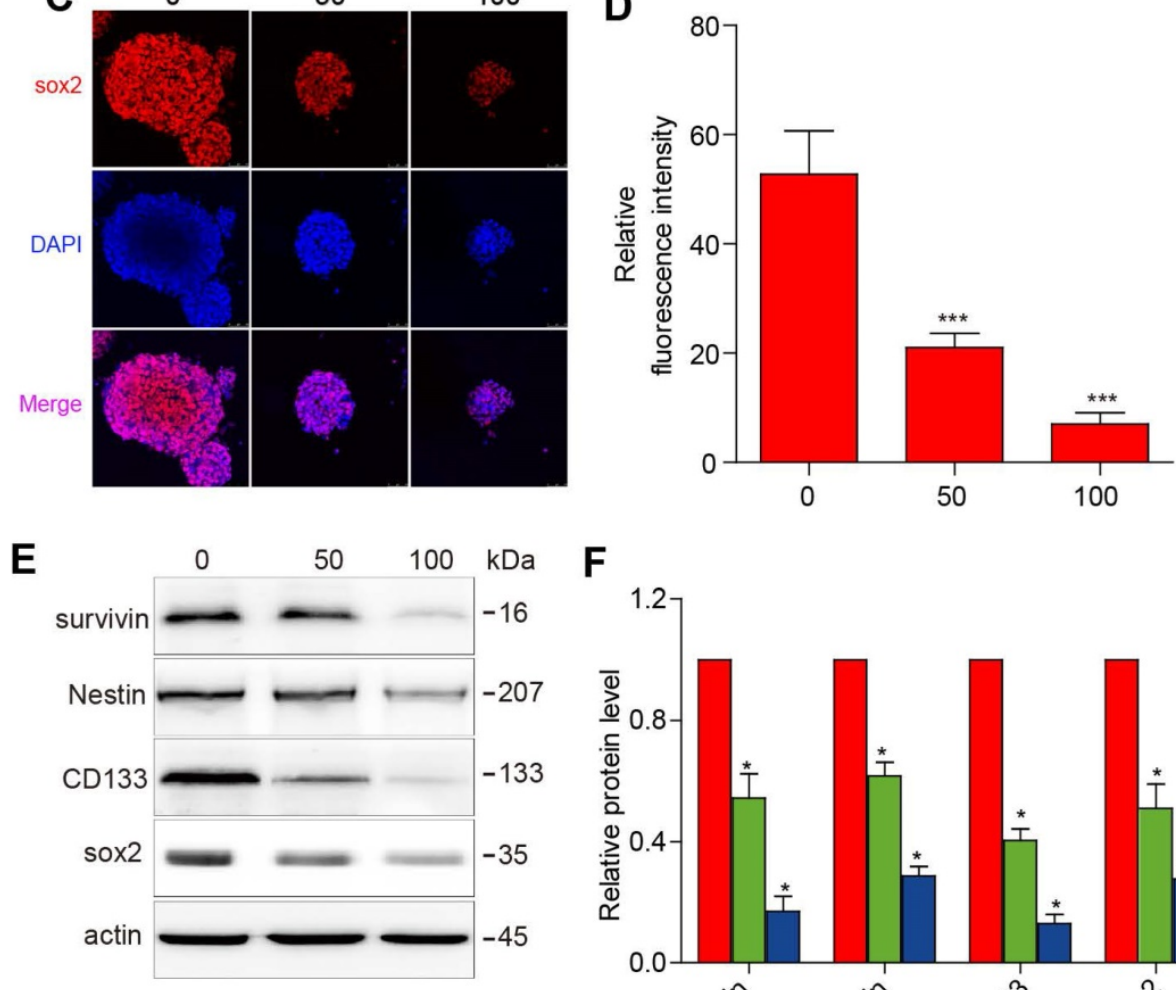

$\mathbf{F}$

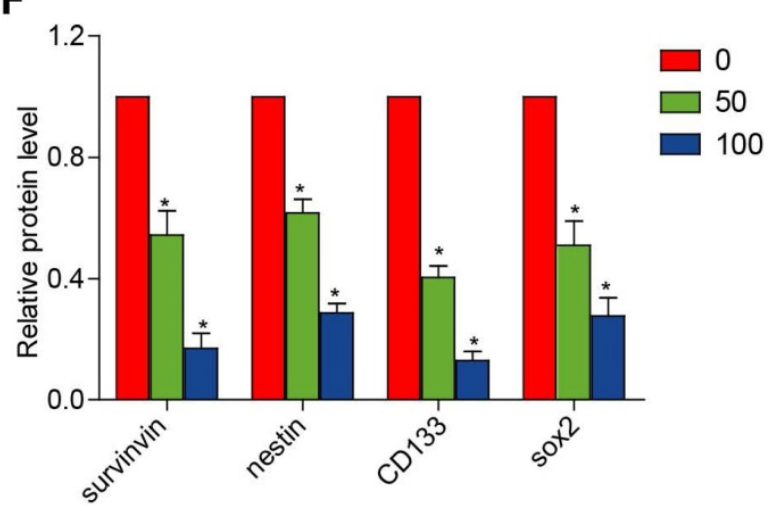

Figure 2. 3BDO inhibits cell growth and downregulates the expression of survivin and stemness markers in GSCs. GSCs were cultured with selected doses of $3 \mathrm{BDO}$ for $72 \mathrm{~h}$ and were then subjected to (A-B) quantification of the spheres formed, (C-D) immunofluorescence examination to determine sox 2 expression levels, (E-F) western blotting analysis to determine protein expression levels of stemness markers and the gels were analyzed. The GSC sphere (White arrow). $n=3$ or $n=4$ in each group. All experiments were performed in triplicate. *, $\mathrm{P}<0.05 ; * *, \mathrm{P}<0.01$; ***, $\mathrm{P}<0.001$, compared with the control $(0 \mu \mathrm{M})$.

Multiple studies have reported that survivin is abundantly expressed abundantly in GBM tissues and also in human-derived GSC cultures. The downregulation of survivin is abolished both in vivo and in vitro during GSC growth. To further investigate whether 3BDO inhibited GSCs through its effect on survivin, we translocated the cells with a survivin overexpressing plasmid or its vector plasmid and subsequently incubated the cells with 3BDO. We found that the overexpression of survivin enhanced the number of spheres formed and upregulated stemness markers, such as nestin, CD133, and sox2. Moreover, the overexpression of survivin reduced the effect of $3 \mathrm{BDO}$ on sphere formation and the upregulation of stemness markers in the GSCs (Figure $4)$. These results suggest that $3 \mathrm{BDO}$ exerts an anti-tumor effect by suppressing survivin-triggered
EMT in GBM cells and by exerting an anti-stemness effect on GSCs.

\section{BDO suppressed GBM growth in a U87 xenograft mouse model}

$3 \mathrm{BDO}$ treatment $(80 \mathrm{mg} / \mathrm{kg} /$ day $)$ over a period of 25 days resulted in significantly lower tumor weight and volume (Figure $5 \mathrm{~A}-\mathrm{C}$ ), without affecting the bodyweight of the mice (Figure 5D), compared with the untreated groups. Additionally, we confirmed that the expression of endogenous survivin, snail, N-cadherin, vimentin, and GSC markers, such as nestin, sox2, and CD133 in tumors dissected from the U87 xenograft mice were suppressed by 3BDO (Figure 5E-F). These findings are in agreement with the in vitro findings, suggesting that 3BDO suppresses tumor growth by inhibiting survivin-mediated EMT and stemness in glioblastoma 
xenograft models, thereby highlighting its potential as a therapeutic GBM candidate. Moreover, in order to detect the liver and kidney toxicity of the drug, when the end of the experiment was reached, we took the kidney and liver of the mouse and stained the HE section. It was found that the liver and kidney of the mice in the control group and the 3BDO group did not occur damage (Figure 5G).

A

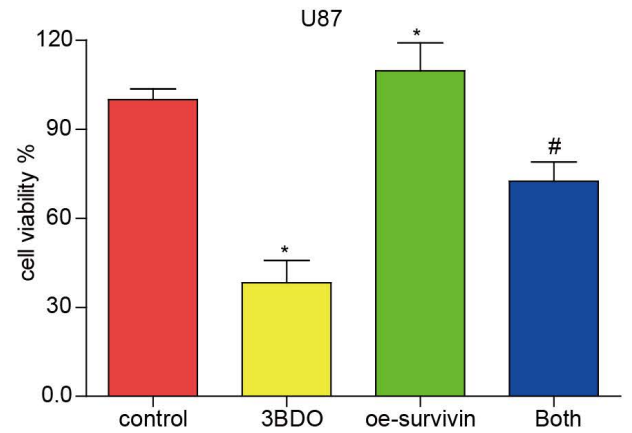

C

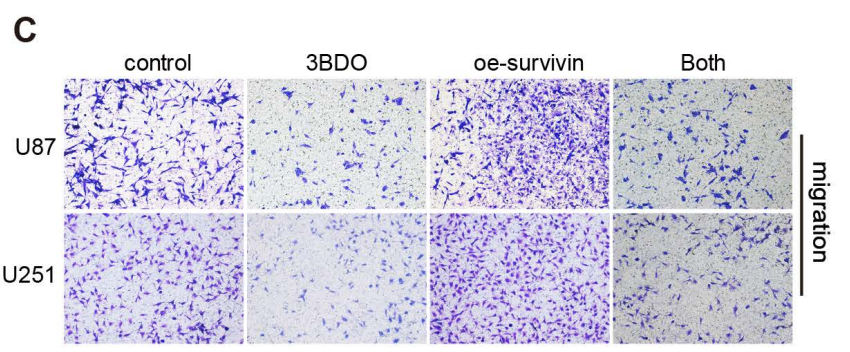

D
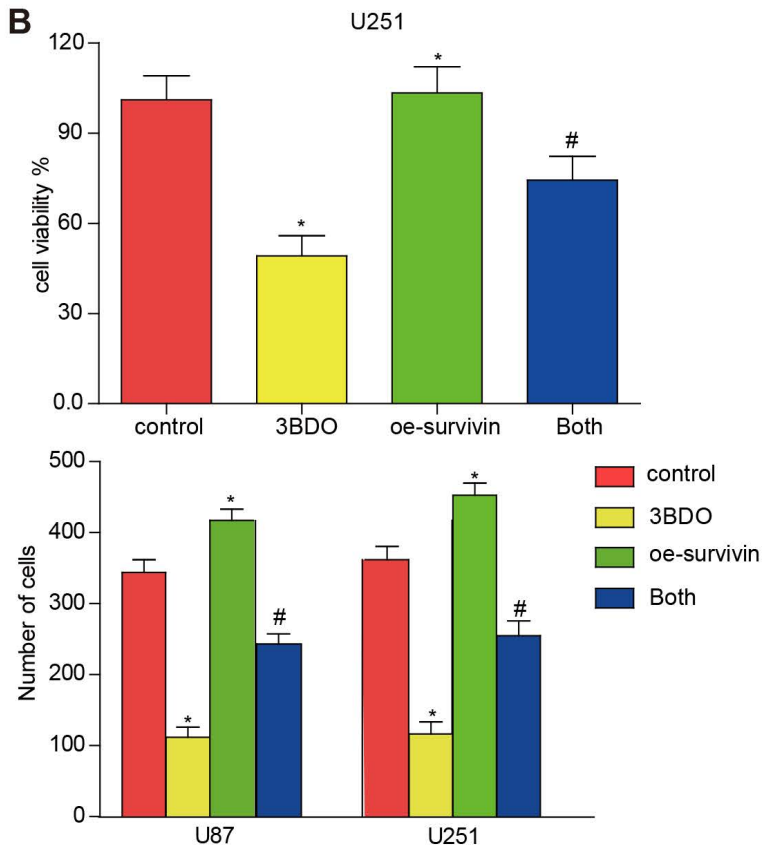

E

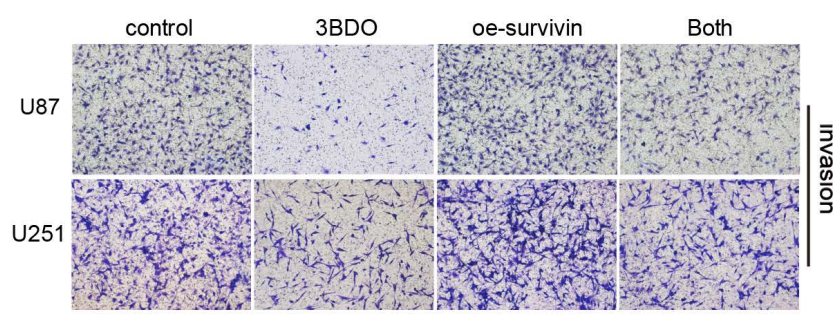

$\mathbf{F}$

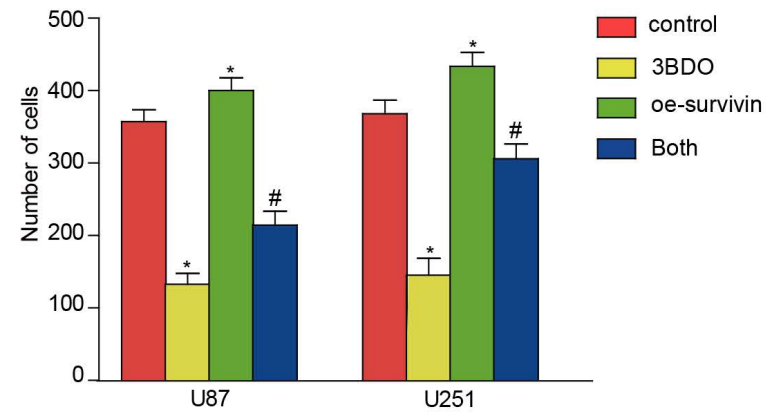

I

\section{Discussion}

Despite the advent of curative GBM therapy that involves the combination of surgical resection and chemoradiotherapy, GBM patient prognosis is still ability. Therefore, novel treatment agents need to be urgently investigated. This study presents 3BDO, a known mTOR activator, as a likely EMT suppressor in GSC cells that exerts its effects through survivin downregulation. abysmal due to its high recurrence and metastatic
G

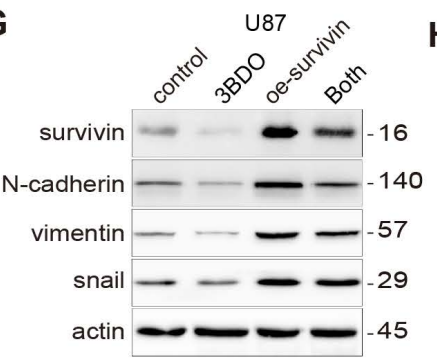

H

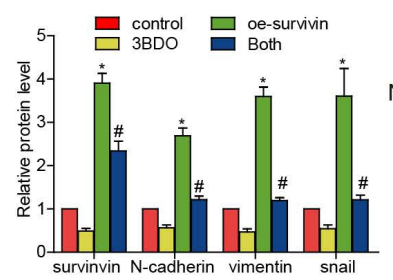

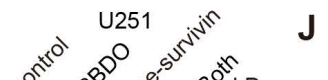
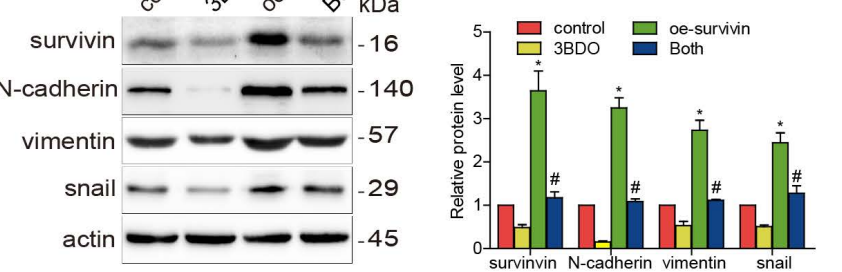

Figure 3. Overexpression of survivin decreases the inhibition effect on cell proliferation, migration, invasion, and EMT induced by 3BDO. (A-B) GBM cells were transfected with survivin or the vector plasmid for 12 hours before being transferred onto 96 -well plates and were treated with $100 \mu M 3 B D O$ or the vehicle for 24 hours. Cellular proliferation was evaluated using a CCK-8 assay. (C-F) Migration and invasion assays were performed on transfected GBM cells using a Transwell assay. (G-J) GBM cells transfected with survivin, or the vector plasmid were treated with $100 \mu \mathrm{M} 3 \mathrm{BDO}$ for $24 \mathrm{~h}$. Western blotting analysis was performed to detect protein expression levels, and the gels were analyzed. *, $\mathrm{P}<0.05$ vs. control; \#, $\mathrm{P}<0.05$, compared with either $3 \mathrm{BDO}$ incubation or survivin transfection alone. 


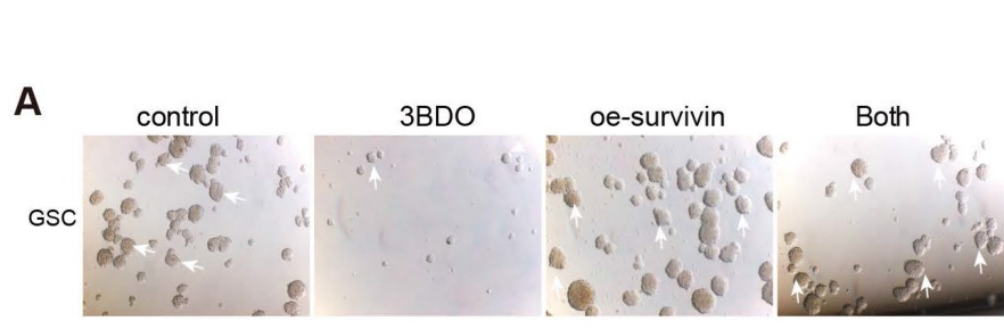

C
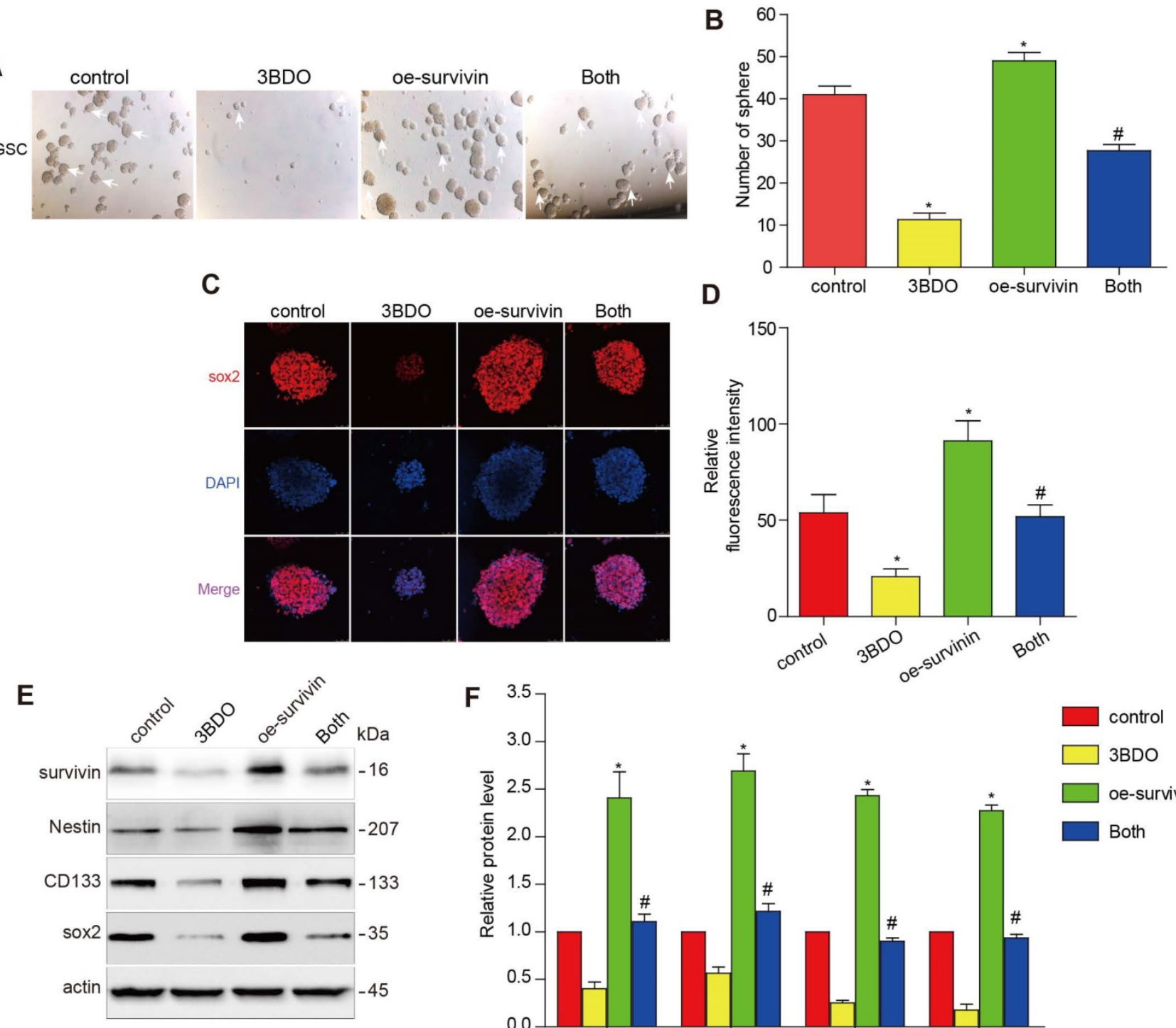

D
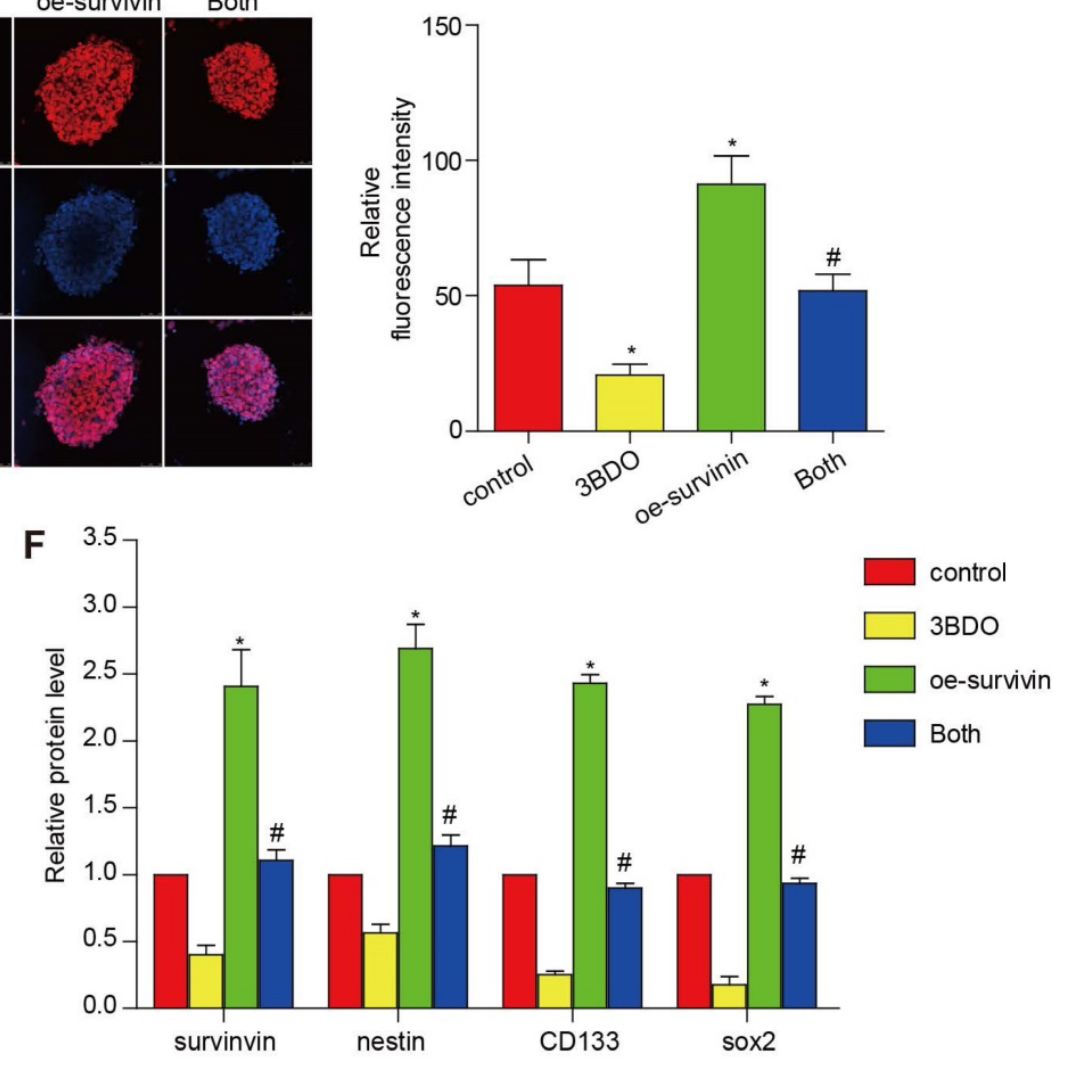

Figure 4. Overexpression of survivin decreases the impact of 3 BDO on GSCs. GSCs were transfected with survivin or the vector plasmid for 12 hours and then cultured with the $100 \mu \mathrm{M} 3 \mathrm{BDO}$. Additionally, the GSCs were incubated with or without $100 \mu \mathrm{M} 3 \mathrm{BDO}$ for $72 \mathrm{~h}$ and then subjected to, (A-B) quantification of the spheres formed (C-D) immunofluorescence examination to determine sox 2 expression levels, (E-F) western blotting analysis to determine protein expression levels of the stemness markers and the gels were cropping. The GSC sphere (White arrow). $\mathrm{n}=3$ or $\mathrm{n}=4$ in each group. All experiments were performed in triplicate. *, $\mathrm{P}<0.05$; $* *, \mathrm{P}<0.01$; $* * *, \mathrm{P}<$ 0.001 , compared with the control $(0 \mu \mathrm{M})$.

3-benzyl-5-((2-nitrophenoxy) methyl)-dihydro furan-2(3H)-one (3BDO), a novel mTOR activator, has demonstrated a variety of biological activity in addition to autophagy suppression [25, 26]. It has been reported that $3 \mathrm{BDO}$ can improve cognitive defects by regulating autophagy in APP/PS1 AD mice models [19]. In addition, 3BDO may be beneficial for the treatment of cardiovascular diseases through autophagy suppression [27]. Moreover, 3BDO could inhibit the production of inflammatory cytokines both in vivo and in vitro [22]. However, the effects of 3BDO in cancer have seldom been reported. Zhao et al. showed that 3BDO in combination with heat shock protein 90 (HSP90) inhibitor 4-(3-(7-(diethylamino)-2oxo-2H-chromen-3-yl)-5-phenyl-4,5-dihydro-1H-pyra zol-1-yl) benzoic acid (DPB) could promote the effectiveness of A549 cell apoptosis [23]. This study explored for the first time the inhibitory effects of $3 \mathrm{BDO}$ on GBM and GCS cells.

EMT has been proven to be closely involved in tumor development. It exerts its effects by maintaining tumor stemness, enhancing drug resistance, and enhancing cellular invasiveness - all of which greatly enhance tumor growth [28]. GSCs are infamous for their high levels of resistance towards chemoradiotherapy and their potent ability to initiate tumor growth $[6,8]$. Therefore, it is essential to identify novel methods of treatment that can inhibit EMT and GSCs for the treatment of GBM. Several agents have been identified as EMT suppressors and 
are able to inhibit GSCs. In this study, we proved that 3BDO exerted anti-invasive and anti-migratory properties, which may reverse EMT progression in GBM. Moreover, we demonstrated that 3BDO inhibited GSC cell growth by suppressing stemness.

Survivin has been shown to be significantly upregulated in GBM and may play a role in promoting GBM development. GBM patients with higher survivin expression had shorter survival times compared with patients with lower survivin expression. In addition, EMT regulation has been reported to be mediated by survivin in GBM.

Liu et al. reported that IGF-1 triggered EMT in hepatocellular carcinoma by activating survivin [29]. Additionally, Lee et al. showed that TGF- $\beta$ regulated the EMT process by upregulating survivin [30]. It has also been reported that the silencing of survivin expression could suppress EMT activation to decrease the invasive and migratory abilities of HCC cells [31]. Knockdown of survivin results in inhibition of epithelial to mesenchymal transition in retinal pigment epithelial cells. Furthermore, Guvenc et al. reported that the suppression of survivin could impair GSC survival [18]. Moreover, brexpiprazole has been shown to promote GSC sensitivity to chemotherapy drugs by downregulating survivin [32]. Therefore, targeting survivin may be a promising strategy for the management of GBM. 3BDO, an autophagy inhibitor, was recently shown to be able to regulate TGFB2 in a manner that does not activate autophagy [22]. In our study, we found that 3BDO could inhibit GBM cells and GSCs via survivin downregulation. Moreover, GBM cells and GSCs overexpressing survivin appeared to develop 3BDO resistance. This suggests that survivin may be a target of 3BDO. However, how 3BDO down-regulates
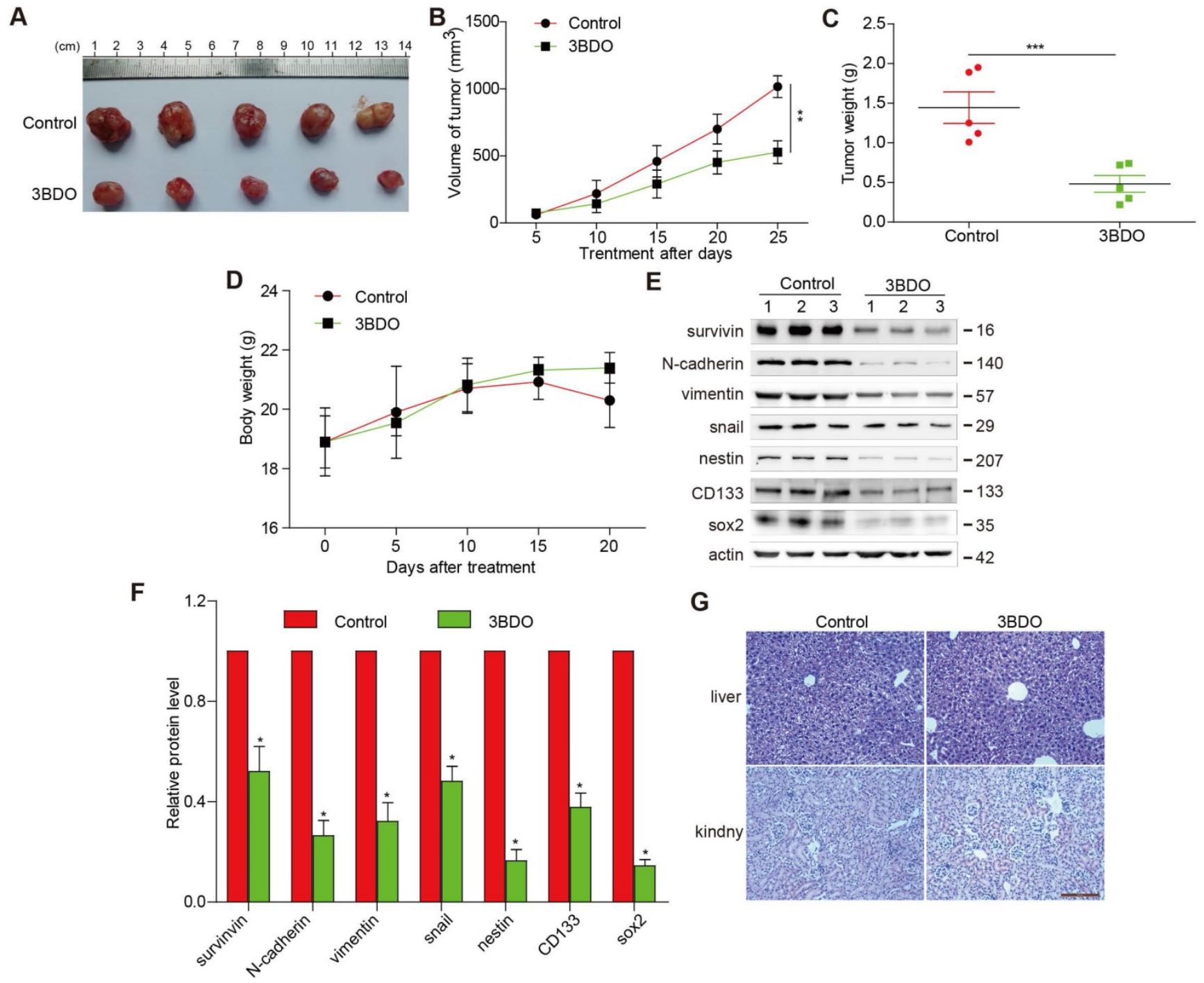

G

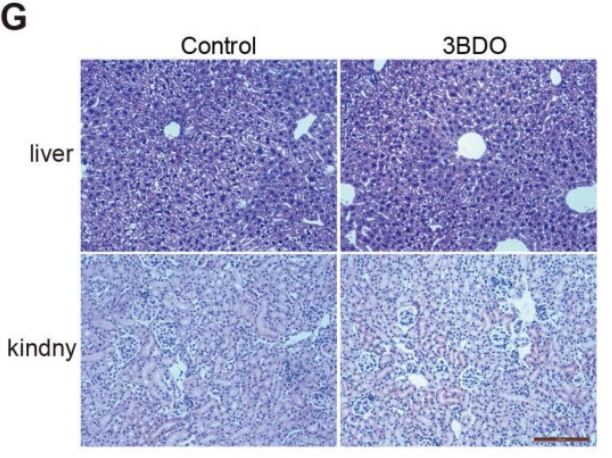

Figure 5. 3BDO decreases glioma growth in a xenograft mouse model. (A) The decrease in the size of xenograft U87 tumors was recorded using photographs (Bar, 1 $\mathrm{cm})$. The tumor volume (B), tumor weight $(C)$, and bodyweight of the mice (D) were measured every 5 days. (E-F) At the end of the experiments, the tumor tissues were excised from the mice, and western blotting analysis was used to detect the protein expression levels, and the gels were analyzed and (G) the kidney and liver tissues were obtained and stained (Bar, $500 \mu \mathrm{m}) . \mathrm{n}=3$ or $\mathrm{n}=4$ in each group. All experiments were performed in triplicate. *, $\mathrm{P}<0.05 ; * *, \mathrm{P}<0.01$; ***, $\mathrm{P}<0.001$, compared with the control $(0 \mu \mathrm{M})$. 
survivin requires further exploration. In addition, it has been reported that activating or inhibiting autophagy by inhibiting or activating the mTOR pathway can inhibit the growth of gliomas by breaking the balance of autophagy. In our research, we found that 3BDO may inhibit the growth of gliomas by acting on survivin. Whether 3BDO inhibits the growth of gliomas by additionally inhibiting autophagy also requires further follow-up investigations.

Most agents that exert an anti-GBM effect in vitro remain ineffective in vivo. Previous drug candidates that have been shown to inhibit tumor growth in a subcutaneous GBM model failed to suppress GBM in the CNS due to the existence of the blood-brain barrier (BBB). Additional experiments evaluating the ability of $3 \mathrm{BDO}$ to inhibit glioma in a mouse stereotactic brain model are needed. Moreover, the safety and efficacy profiles of these drug candidates must be considered, especially for the development of anti-cancer agents. In previous investigations, 3BDO has been used in in vivo experiments, including experiments involving the CNS, and showed a good safety profile [26, 33]. In our study, we found that 3BDO suppressed GBM growth in a subcutaneous GBM model in a manner similar to that demonstrated in our in vitro experiments. The lack of significant changes in overall mice body weight as well as the kidney and liver histopathology confirmed that 3BDO was favorably tolerated.

Overall, we proved that 3BDO successfully suppressed EMT and stemness in GBM both in vivo and in vitro. Additionally, we identified survivin as a potential target of 3BDO. We also demonstrated, for the first time, that 3BDO exerted anti-GBM properties using a subcutaneous glioma model, which was consistent with its mechanism of action demonstrated in the in vitro experiments. Therefore, $3 \mathrm{BDO}$ is a potential therapeutic drug for the treatment of GBM.

\section{Acknowledgements}

\section{Funding}

This work was supported by the National Natural Science Foundation of China (81901117) and the Natural Science Foundation of Guangdong Province (2019A1515010926).

\section{Author Contributions}

Yezhong Wang, Zhou Xing, and Yunxiang Ji designed the study; Zhaotao Wang, Yongping Li, Minyi Liu, and Danmin Chen performed the experiments and prepared the data and graphs; Zhaotao Wang and Yongping $\mathrm{Li}$ contributed to drafting the manuscript. All authors read and approved the final manuscript.

\section{Ethics approval and consent to participate}

The mice were 6-8 weeks old and were reared under institutional animal care guidelines after the experimental procedures involving animals were approved by the Institutional Animal Care Committee of the Institutional Animal Care of the Second Affiliated Hospital of Guangzhou Medical University. Guidelines for the ethical review of laboratory animal welfare (People's Republic of China National Standard GB/T 35892) and ARRIVE guidelines (https://www.nc3rs.org.uk/arriveguideline) were also followed.

\section{Availability of data and materials}

The datasets used and/or analyzed in this study are available from the corresponding author on reasonable request.

\section{Competing Interests}

The authors have declared that no competing interest exists.

\section{References}

[1] Tan A, Ashley D, López G, et al. management of glioblastoma: State of the art and future directions. CA: a cancer journal for clinicians, 2020, 70: 299-312

[2] Lah T, Novak M, Breznik B. Brain malignancies: Glioblastoma and brain metastases. Seminars in cancer biology, 2020, 60: 262-273

[3] Barbato L, Bocchetti M, Di Biase A, et al. Cancer stem cells and targeting strategies. Cells, 2019, 8(8): 926

[4] Jin X, Jin X, Kim H. Cancer stem cells and differentiation therapy. Tumour biology : the journal of the International Society for Oncodevelopmental Biology and Medicine, 2017, 39: 1010428317729933

[5] Miyoshi N, Mizushima T, Doki Y, et al. Cancer stem cells in relation to treatment. Japanese journal of clinical oncology, 2019, 49: 232-237

[6] Lathia J, Mack S, Mulkearns-Hubert E, et al. Cancer stem cells in glioblastoma. Genes \& development, 2015, 29: 1203-1217

[7] Jhaveri N, Chen T, Hofman F. Tumor vasculature and glioma stem cells: Contributions to glioma progression. Cancer letters, 2016, 380: 545-551

[8] Sundar S, Hsieh J, Manjila S, et al. The role of cancer stem cells in glioblastoma. Neurosurgical focus, 2014, 37: E6

[9] Santamaria P, Moreno-Bueno G, Portillo F, et al. Emt: Present and future in clinical oncology. Molecular oncology, 2017, 11: 718-738

[10] Chaffer C, San Juan B, Lim E, et al. Emt, cell plasticity and metastasis. Cancer metastasis reviews, 2016, 35: 645-654

[11] Kahlert U, Nikkhah G, Maciaczyk J. Epithelial-to-mesenchymal(-like) transition as a relevant molecular event in malignant gliomas. Cancer letters, 2013, 331: 131-138

[12] Majc B, Sever T, Zarić M, et al. Epithelial-to-mesenchymal transition as the driver of changing carcinoma and glioblastoma microenvironment. Biochimica et biophysica acta Molecular cell research, 2020, 1867: 118782

[13] $\mathrm{Pu}$ W, Qiu J, Riggins G, et al. Matrix protease production, epithelial-to-mesenchymal transition marker expression and invasion of glioblastoma cells in response to osmotic or hydrostatic pressure. Scientific reports, 2020, 10: 2634

[14] Noh M, Oh S, Ahn E, et al. Prognostic significance of e-cadherin and n-cadherin expression in gliomas. BMC cancer, 2017, 17: 583

[15] Altieri D. The molecular basis and potential role of survivin in cancer diagnosis and therapy. Trends in molecular medicine, 2001, 7: 542-547

[16] Altieri D. Survivin, versatile modulation of cell division and apoptosis in cancer. Oncogene, 2003, 22: 8581-8589

[17] Tong X, Yang P, Wang $K$, et al. Survivin is a prognostic indicator in glioblastoma and may be a target of microrna-218. Oncology letters, 2019, 18: 359-367

[18] Guvenc H, Pavlyukov M, Joshi K, et al. Impairment of glioma stem cell survival and growth by a novel inhibitor for survivin-ran protein complex. Clinical cancer research : an official journal of the American Association for Cancer Research, 2013, 19: 631-642

[19] Wei L, Yang H, Xie Z, et al. A butyrolactone derivative 3bdo alleviates memory deficits and reduces amyloid- $\beta$ deposition in an $a \beta p p / p s 1$ transgenic mouse model. Journal of Alzheimer's disease : JAD, 2012, 30: 531-543 
[20] Wang X, Li M, Gao Y, et al. Rheb1-mtorc1 maintains macrophage differentiation and phagocytosis in mice. Experimental cell research, 2016, 344: 219-228

[21] Meng N, Peng N, Huang S, et al. Heterogeneous nuclear ribonucleoprotein e1 regulates protein disulphide isomerase translation in oxidized low-density lipoprotein-activated endothelial cells. Acta physiologica (Oxford, England), 2015, 213: 664-675

[22] Huang S, Lu W, Ge D, et al. A new microrna signal pathway regulated by long noncoding rna tgfb2-ot1 in autophagy and inflammation of vascular endothelial cells. Autophagy, 2015, 11: 2172-2183

[23] Zhao Y, Li K, Zhao B, et al. Hsp90 inhibitor dpb induces autophagy and more effectively apoptosis in a549 cells combined with autophagy inhibitors. In vitro cellular \& developmental biology Animal, 2019, 55: 349-354

[24] Percie du Sert N, Hurst V, Ahluwalia A, et al. The arrive guidelines 2.0: Updated guidelines for reporting animal research. The Journal of physiology, 2020, 598: 3793-3801

[25] Liu W, Luo H, Wang R, et al. Brapamycin-induced autophagy promotes the chondrogenic differentiation of synovium-derived mesenchymal stem cells in the temporomandibular joint in response to il-1. BioMed research international, 2020, 2020: 4035306

[26] Rakhmanova V, Park S, Lee $S$, et al. 3-benzyl-5-((2-nitrophenoxy) methyl)-dihydrofuran-2(3h)-one suppresses fccri-mediated mast cell degranulation via the inhibition of mtorc2-akt signaling. Biochemical and biophysical research communications, 2020, 521: 72-76

[27] Ge D, Han L, Huang S, et al. Identification of a novel mtor activator and discovery of a competing endogenous rna regulating autophagy in vascular endothelial cells. Autophagy, 2014, 10: 957-971

[28] Saitoh M. Involvement of partial emt in cancer progression. Journal of biochemistry, 2018, 164: 257-264

[29] Liu F, Sun Y, Liu B, et al. Insulin-like growth factor-1 induces epithelial-mesenchymal transition in hepatocellular carcinoma by activating survivin. Oncology reports, 2018, 40: 952-958

[30] Lee J, Choi J, Joo C. Tgf- $\beta 1$ regulates cell fate during epithelial-mesenchymal transition by upregulating survivin. Cell death \& disease, 2013, 4: e714

[31] Le T, Vu T, Ho R, et al. Bevacizumab augments the anti-tumor efficacy of infigratinib in hepatocellular carcinoma. International journal of molecular sciences, 2020, 21(24): 9405

[32] Suzuki S, Yamamoto M, Sanomachi T, et al. Brexpiprazole, a serotonin-dopamine activity modulator, can sensitize glioma stem cells to osimertinib, a third-generation egfr-tki, via survivin reduction. Cancers, 2019, 11(7): 947.

[33] Zhu J, Zou M, Li Y, et al. Absence of trim32 leads to reduced gabaergic interneuron generation and autism-like behaviors in mice via suppressing mtor signaling. Cerebral cortex (New York, NY : 1991), 2020, 30: 3240-3258. 